\title{
Visualisation des figures de cavitation à l'entrée d'un inducteur fretté
}

\author{
Cavitation forms visualisation upstream of a shrouded inducer
}

\author{
par C. Offtinger Brissaud, C. Henry, R. Morel, F. Spettel \\ Equipe Hydraulique INSA de Lyon \\ Laboratoire de Mécanique des Fluides et d'Acoustique \\ E.C.L., U.C.B. Lyon 1, U.M.R. CNRS 5509
}

\begin{abstract}
At part load and with low available NPSH, cavitation induces instabilities in inducers. The visualization of the flow upstream of an inducer through a $500 \mathrm{~mm}$ long clear suction pipe allows us to map the inception and the evolution of the different forms of cavitation appearing upstream of this inducer. The plane NPSHaV $Q$ is explored using two methods. First, the flowrate is reduced at constant NPSHa, it is possible to observe successively blade cavitation then backflow cavitation. The second method consists in lowering the NPSHa at constant flowrate, this brings to the fore the inception of cavitation surge. A great part of load instabilities take place with a head drop minus than $3 \%$.
\end{abstract}

\section{INTRODUCTION}

Un inducteur fretté composé de trois aubes, placé en alimentation d'une pompe centrifuge a été étudié au Laboratoire de Mécanique des Fluides de l'INSA de Lyon [1].

A débit partiel, cet inducteur est le siège d'instabilités de fonctionnement importantes liées à la cavitation. Dans le film présenté, nous nous proposons de montrer les formes de cavitation observables à l'entrée de cet inducteur pour différents points de fonctionnement.

Une présentation détaillée de la machine étudiée ainsi que de la boucle d'essai est réalisée en [2]. Précisons cependant que la vitesse de rotation de l'ensemble inducteur fretté-roue centrifuge est de $2960 \mathrm{tr} / \mathrm{min}$.

L'exploration systématique du plan NPSHdisp/Q, permet de dresser une cartographie où apparaissent des zones caractérisées par de la cavitation d'aubage, un tore de recirculation ou une torche.

\section{M MODE OPÉRATOIRE}

Une manchette transparente permet de visualiser l'écoulement à l'amont et à l'aval immédiat de l'inducteur. Le film est réalisé à l'aide d'une caméra HI 8 (24 images/s) placée perpendiculairement à l'axe de la machine (figure 1).

L'éclairage est réalisé par stroboscopie à une fréquence constante afin de repérer des fluctuations éventuelles de la vitesse de rotation de la roue.

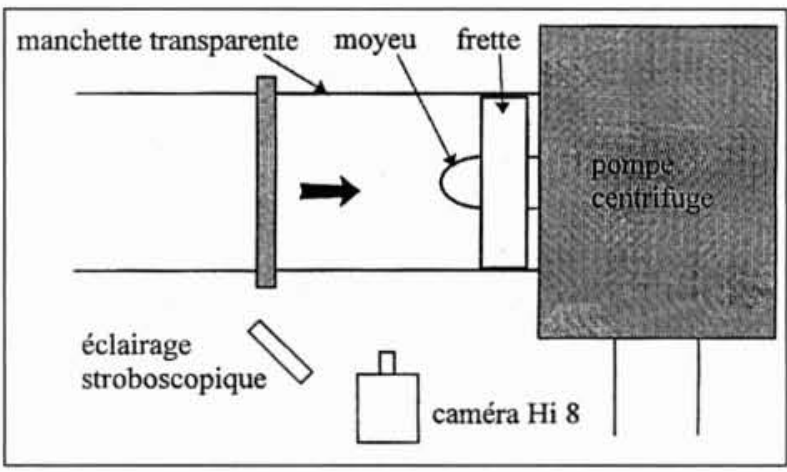

1. Schéma de l'installation.

Le champ de l'image couvre la totalité de la veine à l'amont de l'inducteur jusqu'à une distance d'environ deux diamètres.

Le montage du film a été réalisé à l'aide du logiciel AVID au service audiovisuel de I'INSA de Lyon.

\section{III — VISUALISATION DES FIGURES DE CAVI- TATION}

Le plan NPSHdisp / Q est exploré selon deux procédures. La première consiste à diminuer le débit pour un NPSH disponible donné et permet de mettre en évidence (figure 2) l'apparition et l'évolution de la cavitation d'aubage et de la cavitation de recirculation (tore de recirculation, torche). La 


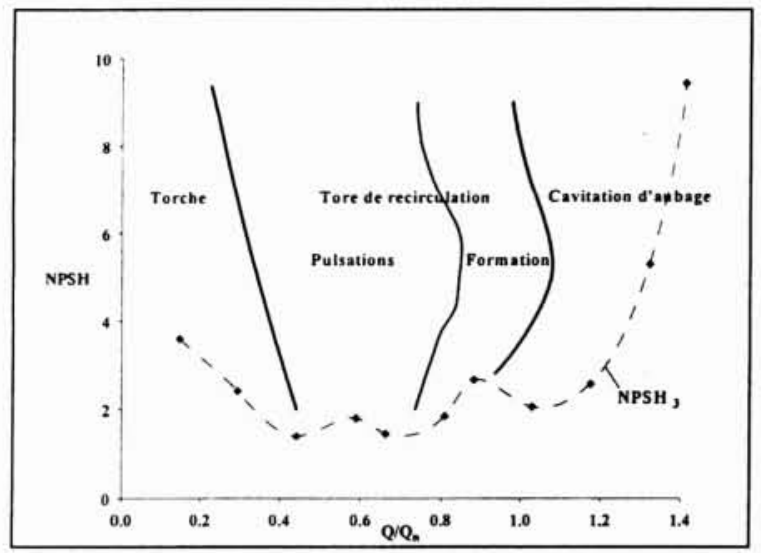

2. Apparition de la cavitation d'aubage.

seconde procédure consiste en des descentes en cavitation à débit fixé ; elle permet de repérer (figure 3) l'apparition de fluctuations en masse des différentes figures de cavitation.

Sur les figures 2 et 3, le débit est rapporté au débit Qn de meilleur rendement de l'ensemble inducteur - roue centrifuge et nous avons tracé à titre indicatif la courbe de NPSH requis à $3 \%$ de chute de hauteur.

La frette n'étant pas transparente, la cavitation d'aubage n'est observable que lorsqu'elle est localisée aux bords d'attaque des aubes.

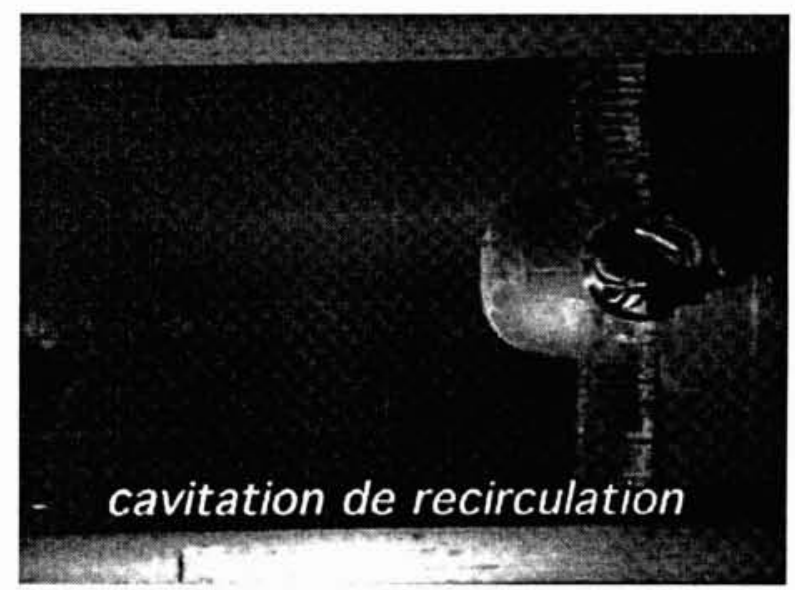

4. Apparition de la cavitation de recirculation.

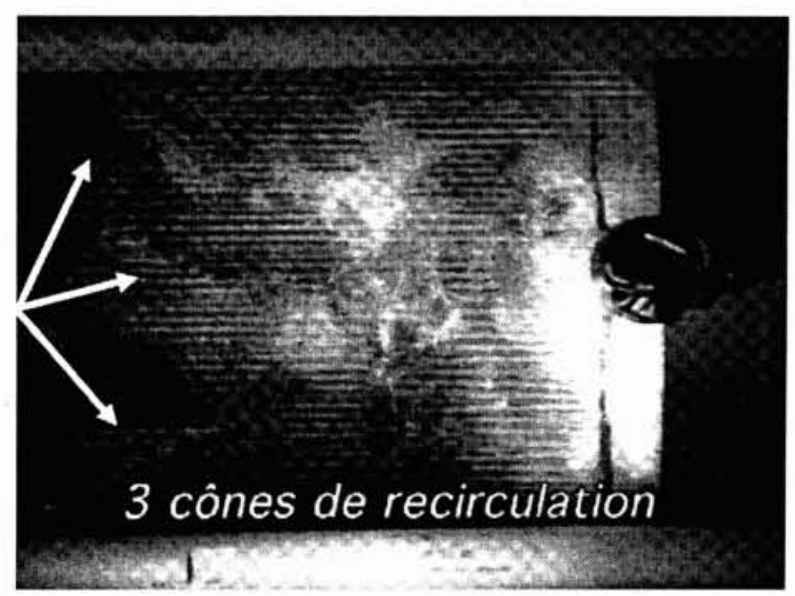

6. Mise en place d'un tore continu de recirculation.

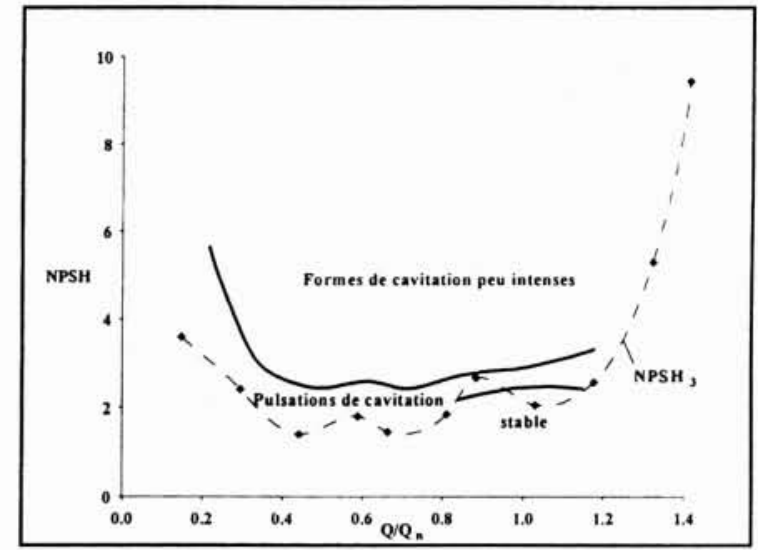

3. Apparition de fluctuations en masse des figures de cavitation.

En diminuant le débit à NPSH disponible fixé, de la cavitation de recirculation apparaît (figure 4) et se développe sous forme de trois cônes séparés desquels s'échappent des petites structures cavitantes isolées appelées filaments fulgurants qui remontent l'écoulement (figure 5). L'étendue axiale des cônes augmente (figure 6) et un tore continu de recirculation se met en place. Des pulsations de la longueur du tore s'établissent progressivement, elles induisent des contraintes sur l'arbre qui se manifestent par des instabilités visibles de la vitesse de rotation. Enfin, pour les très faibles débits

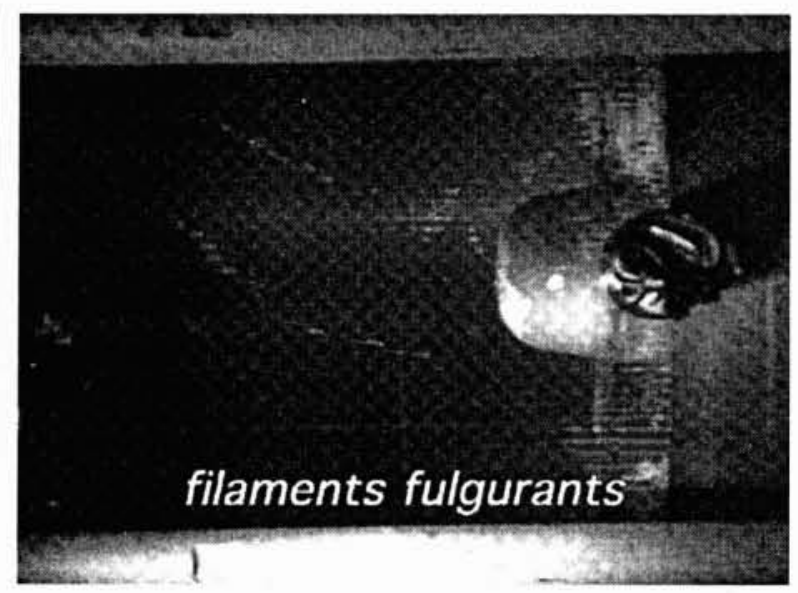

5. Remontée de filaments fulgurants.

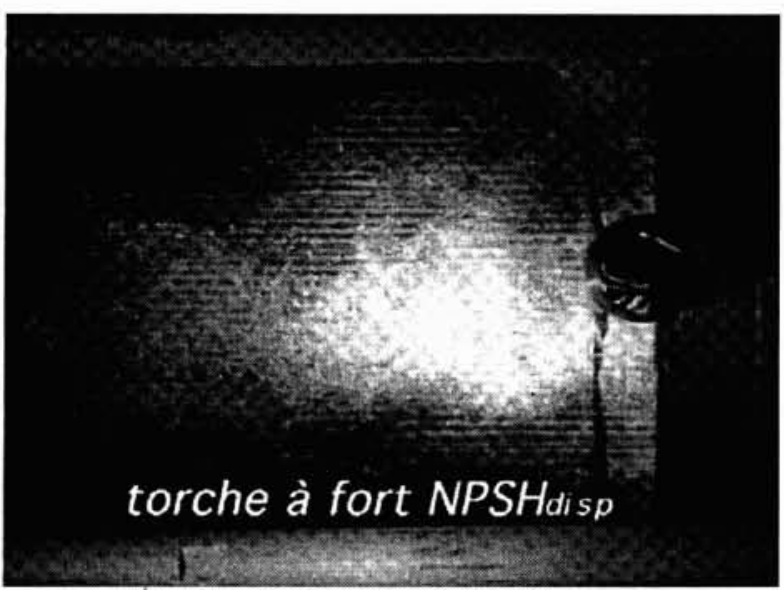

7. Formation d'une torche. 


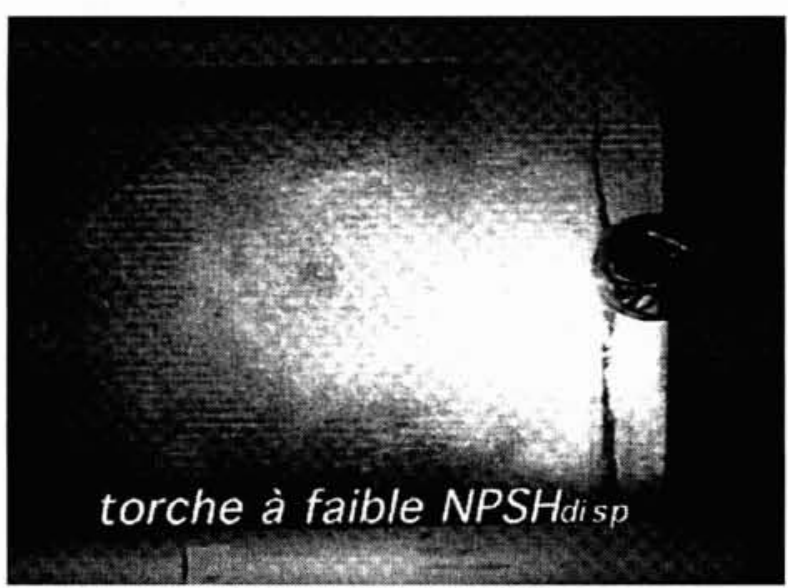

8. Torche à faible NPSH.

$(0,3 \mathrm{Qn})$, la zone amont du tore se contracte vers l'axe de la conduite pour former une torche (figure 7) pulsée.

Les descentes en cavitation à débit constant mettent en évidence l'apparition à faibles NPSH de fluctuations en masse des formes de cavitation décrites précédemment.

Aux débits voisins du débit nominal Qn, ces pulsations basses fréquences s'atténuent pour laisser place à un écoulement presque stable.

A sous-débit, cette atténuation n'est pas obtenue sur la plage de NPSH couverte. A très faible NPSH, la torche occupe quasiment toute la conduite (figure 8) et s'étend périodiquement loin à l'amont de la roue ou disparaît complètement.

Les instabilités de l'écoulement mises en évidence par ces visualisations ont été quantifiées par la mesure des fluctuations de pression pariétale dans les conduites amont et aval ([1], [2]).

\section{IV $\square$ CONCLUSION}

Les prises de vue ont mis en évidence les différentes figures de cavitation se développant à l'amont d'un inducteur fretté. Certaines de ces figures peuvent induire des instabilités de fonctionnement pour des valeurs de NPSH disponibles entraînant une chute de performances inférieure à $3 \%$.

Les variations observées de la vitesse de rotation semblent être la manifestation des contraintes instationnaires importantes sur les aubages.

\section{Remerciements}

Cette étude a été réalisée en collaboration avec EDF DER (Electricité De France - Direction des Etudes et Recherches) et GEC ALSTHOM ACB - BERGERON.

Le montage du film a été effectué avec l'aide de M. Mirallès au service audiovisuel de l'INSA de Lyon.

\section{Bibliographie}

[1] Offtinger Brissaud C. , "Analyse et Réduction des Instabilités de Fonctionnement à Débit Partiel d'un Inducteur Fretté Placé en Alimentation d'une Pompe Centrifuge", thèse de Doctorat de l'INSA de Lyon, Mai 1997. 209 p.

[2] Offtinger C.. Henry C., Morel R., "Instabilités de Fonctionnement à Débit Partiel d'un Inducteur Fretté : Comparaison avec le Cas Non Fretté", La Houille Blanche, $n^{\circ} 4 / 5,1997$, pp. 34-38.

\begin{tabular}{|lll|}
\hline \multicolumn{2}{|c|}{ NOTATIONS : } \\
$\mathrm{Q}$ & $:$ débit volumique \\
$\mathrm{NPSH}$ & $:$ & charge nette à l'aspiration \\
& & \multicolumn{1}{c}{ INDICES : } \\
disp & $:$ & disponible \\
$\mathrm{n}$ & $:$ & nominal (de meilleur rendement) \\
3 & $:$ & requis à $3 \%$ de chute de hauteur \\
\hline
\end{tabular}

